\title{
SUFFICIENCY AND DUALITY FOR INTERVAL-VALUED OPTIMIZATION PROBLEMS WITH VANISHING CONSTRAINTS USING WEAK CONSTRAINT QUALIFICATIONS
}

\author{
IZHAR AHMAD ${ }^{1, *}$, KRISHNA KUMMARI ${ }^{2}$ AND S. AL-HOMIDAN ${ }^{1}$ \\ ${ }^{1}$ Department of Mathematics and Statistics, King Fahd University of Petroleum and Minerals, Dhahran \\ 31261, Saudi Arabia \\ ${ }^{2}$ Department of Mathematics, GITAM-Hyderabad Campus, Hyderabad-502329, India \\ * Corresponding author: drizhar@kfupm.edu.sa

\begin{abstract}
In this paper, we are concerned with one of the difficult class of optimization problems called the interval-valued optimization problem with vanishing constraints. Sufficient optimality conditions for a LU optimal solution are derived under generalized convexity assumptions. Moreover, appropriate duality results are discussed for a Mond-Weir type dual problem. In addition, numerical examples are given to support the sufficient optimality conditions and weak duality theorem.
\end{abstract}

\section{INTRODUCTION}

Due to the mathematical challenges and important roles in various fields, mathematical programs with vanishing constraints have attracted many mathematicians in the past decade. Mathematical programming problem with vanishing constraints is a constrained optimization problem and it is closely related to the Mathematical programs with equilibrium constraints, see for example $[9,10,14]$. This problem was first studied by Achtziger and Kanzow in [2] and this serves as a model for many problems from topology and structural optimization (see $[2,5]$ ). For Mathematical programming problems with vanishing constraints,

Received October $14^{\text {th }}, 2019$; accepted November $13^{\text {th }}$, 2019; published July $17^{\text {th }}, 2020$.

2010 Mathematics Subject Classification. 26A51, 49J35, 90C32.

Key words and phrases. interval-valued optimization problem; vanishing constraints; constraint qualifications; generalized convexity; sufficiency; duality.

(C)2020 Authors retain the copyrights of their papers, and all open access articles are distributed under the terms of the Creative Commons Attribution License. 
it is well known that the usual nonlinear programming constraint qualifications such as Slater constraint qualification, Mangasarian-Fromovitz constraint qualification, Cottle constraint qualification and linear independence constraint qualification do not hold (see [12]), while Mishra et al. [12] proved that the standard generalized Guignard constraint qualification holds in many situations, and some sufficient conditions are presented in [12].

The Guignard constraint qualification (GCQ) was introduced by Guignard [8] and is one of the weakest among the most prominent constraint qualifications such as the Slater constraint qualification [19], Abadie constraint qualification [1], Mangasarian-Fromovitz constraint qualification [11], Cottle constraint qualification [7] and linear independence constraint qualification etc. For more information and inter-relation between these constraint qualifications one can see the survey papers [15,22].

In recent years, a number of approaches have been developed to deal with interval-valued optimization problems. In [24, 25], Wu derived Karush-Kuhn-Tucker type optimality conditions for a optimization problem with an interval-valued objective function. Further, the Karush- Kuhn-Tucker type necessary optimality conditions for a optimization problem in which objective and constraints functions are assumed to be interval valued were investigated by Singh et al. [17]. However, optimality conditions for an interval-valued multiobjective programming with generalized differentiable functions (viz. $g H$-differentiable functions) are discussed in [18]. Bhurjee and Panda [6] provided an overview of an interval-valued optimization problem by developing a methodology to study the efficient solution for an interval-valued optimization problem. For more details related to interval-valued optimization problems, we refer to the papers (see, for example $[3,13,16,20,23,26]$ ). To the author's knowledge, there are no results for an interval-valued mathematical programming problem with vanishing constraints in the literature. Therefore, this paper focuses on an interval-valued mathematical programming problem with vanishing constraints to explore the sufficient optimality conditions and Mond-Weir type duality results.

The rest of the article is organized as follows: Some background material and preliminary definitions are provided in Section 2. The sufficient optimality conditions for a LU optimal solution for considered problem under generalized convexity assumptions are given in Section 3. In Section 4, weak, strong and strict converse duality theorems are discussed for a Mond-Weir type dual model. Finally, Section 5 is devoted to the conclusion.

\section{Preliminaries}

For a nonempty subset $Q$ of $R^{n}$, we use the notations $\operatorname{cl} Q$ and $\operatorname{clco} Q$ to denote the closure of $Q$ and closure of the convex hull of $Q$, respectively. Let $\Theta$ be the set of all closed and bounded intervals in $R$. Let $\Theta_{1}=\left[\tau^{L}, \tau^{U}\right], \Theta_{2}=\left[\rho^{L}, \rho^{U}\right] \in \Theta$, then we have

(i) $\Theta_{1}+\Theta_{2}=\left\{\tau+\rho \mid \tau \in \Theta_{1}\right.$ and $\left.\rho \in \Theta_{2}\right\}=\left[\tau^{L}+\rho^{L}, \tau^{U}+\rho^{U}\right]$ 
(ii) $-\Theta_{1}=\left\{-\tau \mid \tau \in \Theta_{1}\right\}=\left[-\tau^{U},-\tau^{L}\right]$,

(iii) $\Theta_{1}-\Theta_{2}=\Theta_{1}+\left(-\Theta_{2}\right)=\left[\tau^{L}-\rho^{U}, \tau^{U}-\rho^{L}\right]$,

(iv) $k+\Theta_{1}=\left\{k+\tau \mid \tau \in \Theta_{1}\right\}=\left[k+\tau^{L}, k+\tau^{U}\right]$

(v) $k \Theta_{1}=\left\{k \tau \mid \tau \in \Theta_{1}\right\}=\left\{\begin{array}{l}{\left[k \tau^{L}, k \tau^{U}\right], \text { if } k \geq 0,} \\ {\left[k \tau^{U}, k \tau^{L}\right], \text { if } k<0,}\end{array}\right.$

where $k$ is a real number.

For $\Theta_{1}=\left[\tau^{L}, \tau^{U}\right]$ and $\Theta_{2}=\left[\rho^{L}, \rho^{U}\right]$, the order relation $\leq_{L U}$ is defined as follows:

(i) $\Theta_{1} \leq_{L U} \Theta_{2}$ if and only if $\tau^{L} \leq \rho^{L}$ and $\tau^{U} \leq \rho^{U}$.

(ii) $\Theta_{1}<_{L U} \Theta_{2}$ if and only if $\Theta_{1} \leq_{L U} \Theta_{2}$ and $\Theta_{1} \neq \Theta_{2}$.

It is obvious that, $\Theta_{1}<_{L U} \Theta_{2}$ if and only if

$$
\begin{aligned}
& \tau^{L}<\rho^{L} \text { and } \tau^{U}<\rho^{U}, \\
& \text { or, } \quad \tau^{L} \leq \rho^{L} \text { and } \tau^{U}<\rho^{U}, \\
& \text { or, } \quad \tau^{L}<\rho^{L} \text { and } \tau^{U} \leq \rho^{U} .
\end{aligned}
$$

An interval-valued function $\Psi: R^{n} \rightarrow \Theta$ can be written as $\Psi(x)=\left[\Psi^{L}(x), \Psi^{U}(x)\right]$, where $\Psi^{L}(x), \Psi^{U}(x)$ are real-valued functions satisfying the condition $\Psi^{L}(x) \leq \Psi^{U}(x)$ and $\Theta$ be the set of all closed and bounded intervals in $R$. In the present analysis, we consider the following inter-valued optimization problem with vanishing constraints:

(IVVC)

$$
\min _{x \in \mathbb{F}} \Psi(x)=\left[\Psi^{L}(x), \Psi^{U}(x)\right]
$$

subject to

$$
\begin{gathered}
\varphi_{i}(x) \leq 0, \forall i=1,2, \ldots, p, \\
\zeta_{i}(x)=0, \forall i=1,2, \ldots, q, \\
\ell_{i}(x) \geq 0, \forall i=1,2, \ldots, r, \\
\Phi_{i}(x) \ell_{i}(x) \leq 0, \forall i=1,2, \ldots, r,
\end{gathered}
$$

where $\Psi: R^{n} \rightarrow \Theta$ is an interval-valued function and $\Psi^{L}, \Psi^{U}, \varphi_{i}, \zeta_{i}, \ell_{i}, \Phi_{i}: R^{n} \rightarrow R$ are assumed to be continuously differentiable functions. The feasible region is given by

$$
\begin{gathered}
\mathbb{F}=\left\{x \in R^{n} \mid \varphi_{i}(x) \leq 0, \forall i=1,2, \ldots, p,\right. \\
\zeta_{i}(x)=0, \forall i=1,2, \ldots, q, \\
\ell_{i}(x) \geq 0, \forall i=1,2, \ldots, r, \\
\left.\Phi_{i}(x) \ell_{i}(x) \leq 0, \forall i=1,2, \ldots, r\right\} .
\end{gathered}
$$


Definition 2.1 (Sun and Wang [21]). A point $\bar{a} \in \mathbb{F}$ is said to be a LU optimal solution to (IVVC), if there exists no $x_{0} \in \mathbb{F}$ such that $\Psi\left(x_{0}\right)<_{L U} \Psi(\bar{a})$.

Let $x^{*} \in \mathbb{F}$ be any feasible solution of the (IVVC). The following index sets will be used in the sequel.

$$
\begin{aligned}
& \Lambda_{\varphi}=\left\{i \in\{1,2, \ldots, p\} \mid \varphi_{i}\left(x^{*}\right)=0\right\}, \\
& \Lambda_{\zeta}=\{1,2, \ldots, q\}, \\
& \Lambda_{+}=\left\{i \in\{1,2, \ldots, r\} \mid \ell_{i}\left(x^{*}\right)>0\right\}, \\
& \Lambda_{0}=\left\{i \in\{1,2, \ldots, r\} \mid \ell_{i}\left(x^{*}\right)=0\right\} .
\end{aligned}
$$

Furthermore, the index set $\Lambda_{+}$can be divided into the following subsets

$$
\begin{aligned}
& \Lambda_{+0}=\left\{i \in\{1,2, \ldots, r\} \mid \ell_{i}\left(x^{*}\right)>0, \Phi_{i}\left(x^{*}\right)=0\right\}, \\
& \Lambda_{+-}=\left\{i \in\{1,2, \ldots, r\} \mid \ell_{i}\left(x^{*}\right)>0, \Phi_{i}\left(x^{*}\right)<0\right\} .
\end{aligned}
$$

Similarly, the index set $\Lambda_{0}$ can be partitioned in the following way

$$
\begin{aligned}
& \Lambda_{0+}=\left\{i \in\{1,2, \ldots, r\} \mid \ell_{i}\left(x^{*}\right)=0, \Phi_{i}\left(x^{*}\right)>0\right\}, \\
& \Lambda_{00}=\left\{i \in\{1,2, \ldots, r\} \mid \ell_{i}\left(x^{*}\right)=0, \Phi_{i}\left(x^{*}\right)=0\right\}, \\
& \Lambda_{0-}=\left\{i \in\{1,2, \ldots, r\} \mid \ell_{i}\left(x^{*}\right)=0, \Phi_{i}\left(x^{*}\right)<0\right\} .
\end{aligned}
$$

Also, for $x^{*} \in \mathbb{F}$, we define the sets $Q^{k}, \bar{Q}^{k}, k=L, U$ and $\bar{Q}$ as follows:

$$
\begin{gathered}
Q^{k}=\left\{x \in R^{n} \mid \Psi^{i}(x) \leq \Psi^{i}\left(x^{*}\right), \forall i=L, U, i \neq k,\right. \\
\varphi_{i}(x) \leq 0, \forall i=1,2, \ldots, p, \\
\zeta_{i}(x)=0, \forall i=1,2, \ldots, q, \\
\ell_{i}(x) \geq 0, \forall i=1,2, \ldots, r, \\
\left.\Phi_{i}(x) \ell_{i}(x) \leq 0, \forall i=1,2, \ldots, r\right\} . \\
\bar{Q}^{k}=\left\{x \in R^{n} \mid \Psi^{i}(x) \leq \Psi^{i}\left(x^{*}\right), \forall i=L, U, i \neq k\right. \\
\varphi_{i}(x) \leq 0, \forall i=1,2, \ldots, p, \\
\zeta_{i}(x)=0, \forall i=1,2, \ldots, q, \\
\ell_{i}(x)=0, \Phi_{i}(x) \geq 0, \forall i \in \Lambda_{0+}, \\
\left.\Phi_{i}(x) \leq 0, \ell_{i}(x) \geq 0, \forall i \in \Lambda_{0-} \cup \Lambda_{00} \cup \Lambda_{+0} \cup \Lambda_{+-}\right\}
\end{gathered}
$$

and

$$
\bar{Q}=\left\{x \in R^{n} \mid \Psi^{L}(x) \leq \Psi^{L}\left(x^{*}\right), \Psi^{U}(x) \leq \Psi^{U}\left(x^{*}\right),\right.
$$




$$
\begin{gathered}
\varphi_{i}(x) \leq 0, \forall i=1,2, \ldots, p, \\
\zeta_{i}(x)=0, \forall i=1,2, \ldots, q, \\
\ell_{i}(x)=0, \Phi_{i}(x) \geq 0, \forall i \in \Lambda_{0+}, \\
\left.\Phi_{i}(x) \leq 0, \ell_{i}(x) \geq 0, \forall i \in \Lambda_{0-} \cup \Lambda_{00} \cup \Lambda_{+0} \cup \Lambda_{+-}\right\} .
\end{gathered}
$$

The linearizing cone $\bar{Q}^{k}, k=L, U$ at $x^{*} \in \mathbb{F}$ is given by

$$
\begin{gathered}
\mathbb{L}\left(\bar{Q}^{k} ; x^{*}\right)=\left\{\delta \in R^{n} \mid \nabla \Psi^{i}\left(x^{*}\right)^{T} \delta \leq 0, \forall i=L, U, i \neq k,\right. \\
\nabla \varphi_{i}\left(x^{*}\right)^{T} \delta \leq 0, \forall i \in \Lambda_{\varphi}, \\
\nabla \zeta_{i}\left(x^{*}\right)^{T} \delta=0, \forall i \in \Lambda_{\zeta}, \\
\nabla \ell_{i}\left(x^{*}\right)^{T} \delta=0, \forall i \in \Lambda_{0+}, \\
\nabla \ell_{i}\left(x^{*}\right)^{T} \delta \geq 0, \forall i \in \Lambda_{00} \cup \Lambda_{0-}, \\
\left.\nabla \Phi_{i}\left(x^{*}\right)^{T} \delta \leq 0, \forall i \in \Lambda_{+0} \cup \Lambda_{00}\right\} .
\end{gathered}
$$

and the symbol $T$ denotes the transpose of a matrix. The linearizing cone to $\bar{Q}$ at $x^{*} \in \bar{Q}$, given by.

$$
\mathbb{L}\left(\bar{Q} ; x^{*}\right)=\mathbb{L}\left(\bar{Q}^{L} ; x^{*}\right) \cap \mathbb{L}\left(\bar{Q}^{U} ; x^{*}\right) .
$$

Definition 2.2. The tangent cone to $Q$ at $x^{*} \in c l Q$ is defined by

$$
\mathbb{T}\left(x^{*}\right)=\left\{\delta \in R^{n} \mid \exists\left\{x^{n}\right\} \subseteq \mathbb{F},\left\{t_{n}\right\} \downarrow 0: x^{n} \rightarrow x^{*} \text { and } \frac{x^{n}-x^{*}}{t_{n}} \rightarrow \delta\right\}
$$

The modified Guignard constraint qualification was introduced by Mishra et al. ( [12], Definition 6.14) for a mathematical programming problem with vanishing constraints. From this perspective, we define the modified Guignard constraint qualification (IVVC-GCQ) for an interval-valued optimization problem (IVVC) as follows.

Definition 2.3. The modified Guignard constraint qualification (IVVC-GCQ) is said to holds at $x^{*} \in \mathbb{F}$, if $\mathbb{L}\left(\bar{Q} ; x^{*}\right) \subseteq \operatorname{clco} \mathbb{T}\left(Q^{L} ; x^{*}\right) \cap \operatorname{clco} \mathbb{T}\left(Q^{U} ; x^{*}\right)$.

Mishra et al. [12] proved the Karush-Kuhn-Tucker type necessary optimality conditions for a multiobjective optimization problem with vanishing constraints under modified Guignard constraint qualification. Along the lines of Mishra et al. ( [12] Theorem 6.4), if we set $\mathrm{m}=2$, we acquire the following Karush-KuhnTucker type necessary optimality conditions for (IVVC) as follow: 
Theorem 2.1. Let $x^{*} \in \mathbb{F}$ be a LU optimal solution of (IVVC) such that (IVVC-GCQ) holds at $x^{*}$. Then there exist $0<\lambda^{L}, \lambda^{L} \in R, \mu_{i} \in R_{+}, i=1,2, \ldots, p, \gamma_{i} \in R, i=1,2, \ldots, q$ and $\eta_{i}^{\ell}, \eta_{i}^{\Phi} \in R, i=1,2, \ldots, r$ such that

$$
\begin{gathered}
\lambda^{L} \nabla \Psi^{L}\left(x^{*}\right)+\lambda^{U} \nabla \Psi^{U}\left(x^{*}\right)+\sum_{i=1}^{p} \mu_{i} \nabla \varphi_{i}\left(x^{*}\right)+\sum_{i=1}^{q} \gamma_{i} \nabla \zeta_{i}\left(x^{*}\right) \\
-\sum_{i=1}^{r} \eta_{i}^{\ell} \nabla \ell_{i}\left(x^{*}\right)+\sum_{i=1}^{r} \eta_{i}^{\Phi} \nabla \Phi_{i}\left(x^{*}\right)=0, \\
\varphi_{i}\left(x^{*}\right) \leq 0, \mu_{i} \varphi_{i}\left(x^{*}\right)=0, \forall i=1,2, \ldots, p, \\
\zeta_{i}\left(x^{*}\right)=0, \forall i=1,2, \ldots, q, \\
\eta_{i}^{\ell}=0, i \in \Lambda_{+}, \eta_{i}^{\ell} \geq 0, i \in \Lambda_{00} \cup \Lambda_{0-}, \eta_{i}^{\ell} \text { free, } i \in \Lambda_{0+}, \\
\eta_{i}^{\Phi}=0, i \in \Lambda_{+-} \cup \Lambda_{0-} \cup \Lambda_{0+}, \eta_{i}^{\Phi} \geq 0, i \in \Lambda_{+0} \cup \Lambda_{00}, \\
\eta_{i}^{\ell} \ell_{i}\left(x^{*}\right)=0, \eta_{i}^{\Phi} \Phi_{i}\left(x^{*}\right)=0, \forall i=1,2, \ldots, r .
\end{gathered}
$$

We define the following index sets which will be useful to prove the sufficient optimality conditions and duality results.

$$
\begin{array}{cl}
\Lambda_{\varphi}^{+}=\left\{i \in\{1,2, \ldots, p\} \mid \mu_{i}>0\right\}, & \Lambda_{\zeta}^{+}=\left\{i \in \Lambda_{\zeta} \mid \gamma_{i}>0\right\}, \\
\Lambda_{\zeta}^{-}=\left\{i \in \Lambda_{\zeta} \mid \gamma_{i}<0\right\}, & \Lambda_{+}^{+}=\left\{i \in \Lambda_{+} \mid \eta_{i}^{\ell}>0\right\} \\
\Lambda_{0}^{+}=\left\{i \in \Lambda_{0} \mid \eta_{i}^{\ell}>0\right\}, & \Lambda_{0}^{-}=\left\{i \in \Lambda_{0} \mid \eta_{i}^{\ell}<0\right\}, \\
\Lambda_{0+}^{-}=\left\{i \in \Lambda_{0+} \mid \eta_{i}^{\Phi}<0\right\}, & \Lambda_{00}^{-}=\left\{i \in \Lambda_{00} \mid \eta_{i}^{\Phi}<0\right\}, \\
\Lambda_{+0}^{-}=\left\{i \in \Lambda_{+0} \mid \eta_{i}^{\Phi}<0\right\}, & \Lambda_{00}^{+}=\left\{i \in \Lambda_{00} \mid \eta_{i}^{\Phi}>0\right\}, \\
\Lambda_{00}^{-}=\left\{i \in \Lambda_{00} \mid \eta_{i}^{\Phi}<0\right\}, & \Lambda_{+0}^{+}=\left\{i \in \Lambda_{+0} \mid \eta_{i}^{\Phi}>0\right\}, \\
\Lambda_{+0}^{-}=\left\{i \in \Lambda_{+0} \mid \eta_{i}^{\Phi}<0\right\}, & \Lambda_{0-}^{+}=\left\{i \in \Lambda_{0-} \mid \eta_{i}^{\Phi}>0\right\}, \\
\Lambda_{+-}^{+}=\left\{i \in \Lambda_{+-} \mid \eta_{i}^{\Phi}>0\right\} . &
\end{array}
$$

We now turn our attention to define some well-known concepts of convexity and generalized convexity for a real valued differentiable function (see, for example, [4]). 
Definition 2.4. Let $\Omega: X \subseteq R^{n} \rightarrow R$ be a continuously differentiable function. Then, $\Omega$ is said to be $a$ (strictly) convex at $\left(x \neq x^{*} \in X\right) x^{*} \in X$ if for any $x \in X$, we have

$$
\Omega(x)-\Omega\left(x^{*}\right)(>) \geq\left(x-x^{*}\right)^{T} \nabla \Omega\left(x^{*}\right) .
$$

Definition 2.5. Let $\Omega: X \subseteq R^{n} \rightarrow R$ be a continuously differentiable function. Then, $\Omega$ is said to be $a$ quasiconvex at $x^{*} \in X$ if for any $x \in X$, we have

$$
\Omega(x) \leq \Omega\left(x^{*}\right) \Rightarrow\left(x-x^{*}\right)^{T} \nabla \Omega\left(x^{*}\right) \leq 0,
$$

equivalently

$$
\left(x-x^{*}\right)^{T} \nabla \Omega\left(x^{*}\right)>0 \Rightarrow \Omega(x)>\Omega\left(x^{*}\right) .
$$

Definition 2.6. Let $\Omega: X \subseteq R^{n} \rightarrow R$ be a continuously differentiable function. Then, $\Omega$ is said to be $a$ (strictly) pseudoconvex at $x^{*} \in X$ if for any $x \in X$, we have

$$
\left(x-x^{*}\right)^{T} \nabla \Omega\left(x^{*}\right) \geq 0 \Rightarrow \Omega(x)(>) \geq \Omega\left(x^{*}\right),
$$

equivalently

$$
\Omega(x)(\leq)<\Omega\left(x^{*}\right) \Rightarrow\left(x-x^{*}\right)^{T} \nabla \Omega\left(x^{*}\right)<0 .
$$

\section{Sufficient optimality COnditions}

In this section, we establish sufficient optimality conditions for the problem (IVVC) using the concept of generalized convexity.

Theorem 3.1 (Sufficient optimality conditions). Let $\tilde{x} \in \mathbb{F}$ and there exist $0<\lambda^{L}, \lambda^{U} \in R, \mu_{i} \in R_{+}, i=$ $1,2, \ldots, p, \gamma_{i} \in R, i=1,2, \ldots, q$ and $\eta_{i}^{\ell}, \eta_{i}^{\Phi} \in R, i=1,2, \ldots, r$ such that

$$
\begin{aligned}
& \lambda^{L} \nabla \Psi^{L}(\tilde{x})+\lambda^{U} \nabla \Psi^{U}(\tilde{x})+\sum_{i=1}^{p} \mu_{i} \nabla \varphi_{i}(\tilde{x})+\sum_{i=1}^{q} \gamma_{i} \nabla \zeta_{i}(\tilde{x}) \\
& -\sum_{i=1}^{r} \eta_{i}^{\ell} \nabla \ell_{i}(\tilde{x})+\sum_{i=1}^{r} \eta_{i}^{\Phi} \nabla \Phi_{i}(\tilde{x})=0 \\
& \varphi_{i}(\tilde{x}) \leq 0, \mu_{i} \varphi_{i}(\tilde{x})=0, \forall i=1,2, \ldots, p, \\
& \zeta_{i}(\tilde{x})=0, \forall i=1,2, \ldots, q, \\
& \eta_{i}^{\ell}=0, i \in \Lambda_{+}, \eta_{i}^{\ell} \geq 0, i \in \Lambda_{00} \cup \Lambda_{0_{-}}, \eta_{i}^{\ell} \text { free, } i \in \Lambda_{0+}, \\
& \eta_{i}^{\Phi}=0, i \in \Lambda_{+-} \cup \Lambda_{0-} \cup \Lambda_{0+}, \eta_{i}^{\Phi} \geq 0, i \in \Lambda_{+0} \cup \Lambda_{00},
\end{aligned}
$$




$$
\eta_{i}^{\ell} \ell_{i}(\tilde{x})=0, \eta_{i}^{\Phi} \Phi_{i}(\tilde{x})=0, \forall i=1,2, \ldots, r
$$

Further, assume that $\lambda^{L} \Psi^{L}()+.\lambda^{U} \Psi^{U}($.$) is pseudoconvex at \tilde{x}$ on $\mathbb{F}$ and that $\sum_{i=1}^{p} \mu_{i} \varphi_{i}(),. \zeta_{i}().\left(i \in \Lambda_{\zeta}^{+}\right)$, $-\zeta_{i}().\left(i \in \Lambda_{\zeta}^{-}\right),-\ell_{i}().\left(i \in \Lambda_{+}^{+} \cup \Lambda_{0}^{+}\right), \ell_{i}().\left(i \in \Lambda_{0}^{-}\right),-\Phi_{i}().\left(i \in \Lambda_{0+}^{-} \cup \Lambda_{00}^{-} \cup \Lambda_{+0}^{-}\right), \Phi_{i}().\left(i \in \Lambda_{00}^{+} \cup \Lambda_{0-}^{+} \cup \Lambda_{+0}^{+} \cup\right.$ $\left.\Lambda_{+-}^{+}\right)$are quasiconvex at $\tilde{x}$ on $\mathbb{F}$. Then $\tilde{x}$ is a LU optimal solution of the problem (IVVC).

Proof. Suppose contrary to the result that $\tilde{x}$ is not a LU optimal solution to the problem (IVVC), then by Definition 2.1 there exists $x_{0} \in \mathbb{F}$ such that

$$
\Psi\left(x_{0}\right)<_{L U} \Psi(\tilde{x}) .
$$

That is,

$$
\left\{\begin{array} { c } 
{ \Psi ^ { L } ( x _ { 0 } ) < \Psi ^ { L } ( \tilde { x } ) } \\
{ \Psi ^ { U } ( x _ { 0 } ) < \Psi ^ { U } ( \tilde { x } ) }
\end{array} \text { , or } \left\{\begin{array} { c } 
{ \Psi ^ { L } ( x _ { 0 } ) \leq \Psi ^ { L } ( \tilde { x } ) } \\
{ \Psi ^ { U } ( x _ { 0 } ) < \Psi ^ { U } ( \tilde { x } ) }
\end{array} , \text { or } \left\{\begin{array}{c}
\Psi^{L}\left(x_{0}\right)<\Psi^{L}(\tilde{x}) \\
\Psi^{U}\left(x_{0}\right) \leq \Psi^{U}(\tilde{x})
\end{array} .\right.\right.\right.
$$

Since $\lambda^{L}>0, \lambda^{U}>0$, therefore the above inequalities yield

$$
\lambda^{L} \Psi^{L}\left(x_{0}\right)+\lambda^{U} \Psi^{U}\left(x_{0}\right)<\lambda^{L} \Psi^{L}(\tilde{x})+\lambda^{U} \Psi^{U}(\tilde{x})
$$

which by pseudoconvexity of $\lambda^{L} \Psi^{L}()+.\lambda^{U} \Psi^{U}($.$) at \tilde{x}$ on $\mathbb{F}$, we obtain

$$
\left(x_{0}-\tilde{x}\right)^{T}\left[\lambda^{L} \nabla \Psi^{L}(\tilde{x})+\lambda^{U} \nabla \Psi^{U}(\tilde{x})\right]<0 .
$$

For $x_{0} \in \mathbb{F}, \mu_{i} \in R_{+}, i=1,2, \ldots, p$, we have $\mu_{i} \varphi_{i}\left(x_{0}\right) \leq 0, i=1,2, \ldots, p$, which in view of (3.2) implies that

$$
\sum_{i=1}^{p} \mu_{i} \varphi_{i}\left(x_{0}\right) \leq \sum_{i=1}^{p} \mu_{i} \varphi_{i}(\tilde{x})
$$

which by quasiconvexity of $\sum_{i=1}^{p} \mu_{i} \varphi_{i}($.$) at \tilde{x}$ on $\mathbb{F}$, we get

$$
\left(x_{0}-\tilde{x}\right)^{T} \sum_{i=1}^{p} \mu_{i} \nabla \varphi_{i}(\tilde{x}) \leq 0 .
$$

By similar arguments, we have

$$
\begin{gathered}
\left(x_{0}-\tilde{x}\right)^{T} \nabla \zeta_{i}(\tilde{x}) \leq 0, \forall i \in \Lambda_{\zeta}^{+}, \\
-\left(x_{0}-\tilde{x}\right)^{T} \nabla \zeta_{i}(\tilde{x}) \leq 0, \forall i \in \Lambda_{\zeta}^{-}, \\
-\left(x_{0}-\tilde{x}\right)^{T} \nabla \ell_{i}(\tilde{x}) \leq 0, \forall i \in \Lambda_{+}^{+} \cup \Lambda_{0}^{+}, \\
\left(x_{0}-\tilde{x}\right)^{T} \nabla \ell_{i}(\tilde{x}) \leq 0, \forall i \in \Lambda_{0}^{-}, \\
-\left(x_{0}-\tilde{x}\right)^{T} \nabla \Phi_{i}(\tilde{x}) \leq 0, \forall i \in \Lambda_{0+}^{-} \cup \Lambda_{00}^{-} \cup \Lambda_{+0}^{-}, \\
\left(x_{0}-\tilde{x}\right)^{T} \nabla \Phi_{i}(\tilde{x}) \leq 0, \forall i \in \Lambda_{00}^{+} \cup \Lambda_{0-}^{+} \cup \Lambda_{+0}^{+} \cup \Lambda_{+-}^{+},
\end{gathered}
$$


which by the definition of index sets one has

$$
\left(x_{0}-\tilde{x}\right)^{T}\left[\sum_{i=1}^{q} \gamma_{i} \nabla \zeta_{i}(\tilde{x})-\sum_{i=1}^{r} \eta_{i}^{\ell} \nabla \ell_{i}(\tilde{x})+\sum_{i=1}^{r} \eta_{i}^{\Phi} \nabla \Phi_{i}(\tilde{x})\right] \leq 0 .
$$

On adding (3.7), (3.8) and (3.9), we get

$$
\begin{gathered}
\left(x_{0}-\tilde{x}\right)^{T}\left[\lambda^{L} \nabla \Psi^{L}(\tilde{x})+\lambda^{U} \nabla \Psi^{U}(\tilde{x})+\sum_{i=1}^{p} \mu_{i} \nabla \varphi_{i}(\tilde{x})+\sum_{i=1}^{q} \gamma_{i} \nabla \zeta_{i}(\tilde{x})\right. \\
\left.-\sum_{i=1}^{r} \eta_{i}^{\ell} \nabla \ell_{i}(\tilde{x})+\sum_{i=1}^{r} \eta_{i}^{\Phi} \nabla \Phi_{i}(\tilde{x})\right]<0,
\end{gathered}
$$

which contradicts (3.1). This completes the proof of this theorem.

Now, we verify the sufficient optimality conditions by the following example.

Example 3.1. Consider the following interval-valued optimization problem:

$$
\min _{x \in \mathbb{F}_{1}} \Psi(x)=\left[\Psi^{L}(x), \Psi^{U}(x)\right]=\left[x+x^{3}, x^{5}\right]
$$

subject to

$$
\begin{gathered}
\ell_{1}(x)=1+x^{3} \geq 0, \\
\Phi_{1}(x) \ell_{1}(x)=x\left(1+x^{3}\right) \leq 0,
\end{gathered}
$$

which is the form of (IVVC) with $n=1, p=q=0$ and $r=1$. The feasible region of (IVVC-1) is

$$
\mathbb{F}_{1}=\left\{x \in R \mid \ell_{1}(x) \geq 0, \Phi_{1}(x) \ell_{1}(x) \leq 0\right\}
$$

Note that $\tilde{x}=0$ is a feasible solution of (IVVC-1) and it can be easily observe that there exist $0<\lambda^{L}, \lambda^{U} \in R$, $\eta_{1}^{\ell}$, and $\eta_{1}^{\Phi} \in R$ such that the relations (3.1)-(3.6) hold for the problem (IVVC-1). Also, it is not difficult to see that $\lambda^{L} \Psi^{L}()+.\lambda^{U} \Psi^{U}($.$) is pseudoconvex at \tilde{x}$ on $\mathbb{F}_{1}$ and $\ell_{1}(x), \Phi_{1}(x)$ are quasiconvex at $\tilde{x}$ on $\mathbb{F}_{1}$. Since all the assumptions of Theorem 3.1 are satisfied, then $\tilde{x}=0$ is a LU optimal solution of the problem (IVVC-1).

\section{Mond-Weir type DUALITy}

We present the following Mond-Weir type dual for (IVVC).

(IMWDVC)

$$
\max \Psi(y)=\left[\Psi^{L}(y), \Psi^{U}(y)\right]
$$

subject to

$$
\begin{gathered}
\lambda^{L} \nabla \Psi^{L}(y)+\lambda^{U} \nabla \Psi^{U}(y)+\sum_{i=1}^{p} \mu_{i} \nabla \varphi_{i}(y)+\sum_{i=1}^{q} \gamma_{i} \nabla \zeta_{i}(y) \\
-\sum_{i=1}^{r} \eta_{i}^{\ell} \nabla \ell_{i}(y)+\sum_{i=1}^{r} \eta_{i}^{\Phi} \nabla \Phi_{i}(y)=0
\end{gathered}
$$




$$
\begin{gathered}
\mu_{i} \geq 0, \mu_{i} \varphi_{i}(y) \geq 0, \forall i=1,2, \ldots, p \\
\gamma_{i} \in R, \gamma_{i} \zeta_{i}(y)=0, \forall i=1,2, \ldots, q, \\
\eta_{i}^{\ell} \geq 0, \forall i \in \Lambda_{+}, \eta_{i}^{\ell} \in R, \forall i \in \Lambda_{0}, \\
-\eta_{i}^{\ell} \ell_{i}(y) \geq 0, \forall i=1,2, \ldots, r, \\
0<\lambda^{L}, \lambda^{U} \in R, \eta_{i}^{\Phi} \leq 0, \forall i \in \Lambda_{0+}, \eta_{i}^{\Phi} \geq 0, \forall i \in\left(\Lambda_{0-} \cup \Lambda_{+-}\right), \\
\eta_{i}^{\Phi} \in R, \forall i \in\left(\Lambda_{00} \cup \Lambda_{+0}\right), \eta_{i}^{\Phi} \Phi_{i}(y) \geq 0, \forall i=1,2, \ldots, r .
\end{gathered}
$$

We denote by $\mathbb{W}_{1}$ the set of all feasible solutions of the problem (IMWDVC) and let $p r \mathbb{W}_{1}=\{y \in$ $\left.R^{n} \mid\left(y, \lambda^{L}, \lambda^{U}, \mu, \gamma, \eta^{\ell}, \eta^{\Phi}\right) \in W_{1}\right\}$ be the projection of the set $\mathbb{W}_{1}$ on $R^{n}$

Now, we prove duality results between problems (IVVC) and (IMWDVC) under certain generalized convexity assumptions imposed on the involved functions.

Theorem 4.1 (Weak Duality). Let $x \in \mathbb{F}$ and $\left(y, \lambda^{L}, \lambda^{U}, \mu, \gamma, \eta^{\ell}, \eta^{\Phi}\right) \in \mathbb{W}_{1}$. Further, assume that $\lambda^{L} \Psi^{L}()+$. $\lambda^{U} \Psi^{U}($.$) is pseudoconvex at y$ on $\mathbb{F} \cup p r \mathbb{W}_{1}$ and that $\sum_{i=1}^{p} \mu_{i} \varphi_{i}(),. \zeta_{i}().\left(i \in \Lambda_{\zeta}^{+}\right),-\zeta_{i}().\left(i \in \Lambda_{\zeta}^{-}\right),-\ell_{i}().(i \in$ $\left.\Lambda_{+}^{+} \cup \Lambda_{0}^{+}\right), \ell_{i}().\left(i \in \Lambda_{0}^{-}\right),-\Phi_{i}().\left(i \in \Lambda_{0+}^{-} \cup \Lambda_{00}^{-} \cup \Lambda_{+0}^{-}\right), \Phi_{i}().\left(i \in \Lambda_{00}^{+} \cup \Lambda_{0-}^{+} \cup \Lambda_{+0}^{+} \cup \Lambda_{+-}^{+}\right)$are quasiconvex at $y$ on $\mathbb{F} \cup p r \mathbb{W}_{1}$, then $\Psi(x) \geq_{L U} \Psi(y)$.

Proof. Suppose, contrary to the result, that

$$
\Psi(x)<_{L U} \Psi(y)
$$

That is,

$$
\left\{\begin{array} { l } 
{ \Psi ^ { L } ( x ) < \Psi ^ { L } ( y ) } \\
{ \Psi ^ { U } ( x ) < \Psi ^ { U } ( y ) }
\end{array} , \text { or } \left\{\begin{array} { l } 
{ \Psi ^ { L } ( x ) \leq \Psi ^ { L } ( y ) } \\
{ \Psi ^ { U } ( x ) < \Psi ^ { U } ( y ) }
\end{array} , \text { or } \left\{\begin{array}{l}
\Psi^{L}(x)<\Psi^{L}(y) \\
\Psi^{U}(x) \leq \Psi^{U}(y)
\end{array} .\right.\right.\right.
$$

Since $\lambda^{L}>0, \lambda^{U}>0$, therefore the above inequalities yield

$$
\lambda^{L} \Psi^{L}(x)+\lambda^{U} \Psi^{U}(x)<\lambda^{L} \Psi^{L}(y)+\lambda^{U} \Psi^{U}(y)
$$

which by pseudoconvexity of $\lambda^{L} \Psi^{L}()+.\lambda^{U} \Psi^{U}($.$) at y$ on $\mathbb{F} \cup p r \mathbb{W}_{1}$, we obtain

$$
(x-y)^{T}\left[\lambda^{L} \nabla \Psi^{L}(y)+\lambda^{U} \nabla \Psi^{U}(y)\right]<0 .
$$


For $x \in \mathbb{F}, \mu_{i} \geq 0, i=1,2, \ldots, p$, we have $\mu_{i} \varphi_{i}(x) \leq 0, i=1,2, \ldots, p$, which in view of (4.2) implies that

$$
\sum_{i=1}^{p} \mu_{i} \varphi_{i}(x) \leq \sum_{i=1}^{p} \mu_{i} \varphi_{i}(y)
$$

which by quasiconvexity of $\sum_{i=1}^{p} \mu_{i} \varphi_{i}($.$) at y$ on $\mathbb{F} \cup p r \mathbb{W}_{1}$, we get

$$
(x-y)^{T} \sum_{i=1}^{p} \mu_{i} \nabla \varphi_{i}(y) \leq 0 .
$$

By similar arguments, we have

$$
\begin{gathered}
(x-y)^{T} \nabla \zeta_{i}(y) \leq 0, \forall i \in \Lambda_{\zeta}^{+}, \\
-(x-y)^{T} \nabla \zeta_{i}(y) \leq 0, \forall i \in \Lambda_{\zeta}^{-}, \\
-(x-y)^{T} \nabla \ell_{i}(y) \leq 0, \forall i \in \Lambda_{+}^{+} \cup \Lambda_{0}^{+}, \\
(x-y)^{T} \nabla \ell_{i}(y) \leq 0, \forall i \in \Lambda_{0}^{-}, \\
-(x-y)^{T} \nabla \Phi_{i}(y) \leq 0, \forall i \in \Lambda_{0+}^{-} \cup \Lambda_{00}^{-} \cup \Lambda_{+0}^{-}, \\
(x-y)^{T} \nabla \Phi_{i}(y) \leq 0, \forall i \in \Lambda_{00}^{+} \cup \Lambda_{0-}^{+} \cup \Lambda_{+0}^{+} \cup \Lambda_{+-}^{+},
\end{gathered}
$$

which by the definition of index sets one has

$$
(x-y)^{T}\left[\sum_{i=1}^{q} \gamma_{i} \nabla \zeta_{i}(y)-\sum_{i=1}^{r} \eta_{i}^{\ell} \nabla \ell_{i}(y)+\sum_{i=1}^{r} \eta_{i}^{\Phi} \nabla \Phi_{i}(y)\right] \leq 0 .
$$

On adding (4.8), (4.9) and (4.10), we get

$$
\begin{gathered}
(x-y)^{T}\left[\lambda^{L} \nabla \Psi^{L}(y)+\lambda^{U} \nabla \Psi^{U}(y)+\sum_{i=1}^{p} \mu_{i} \nabla \varphi_{i}(y)+\sum_{i=1}^{q} \gamma_{i} \nabla \zeta_{i}(y)\right. \\
\left.-\sum_{i=1}^{r} \eta_{i}^{\ell} \nabla \ell_{i}(y)+\sum_{i=1}^{r} \eta_{i}^{\Phi} \nabla \Phi_{i}(y)\right]<0
\end{gathered}
$$

which contradicts (4.1). This completes the proof of this theorem.

Now, we verify the weak duality theorem by the following example.

Example 4.1. Consider the following interval-valued optimization problem:

$$
\min _{x \in \mathbb{F}_{2}} \Psi(x)=\left[\Psi^{L}(x), \Psi^{U}(x)\right]=\left[x+x^{3}, x^{5}\right]
$$

subject to

$$
\begin{gathered}
\ell_{1}(x)=x^{3} \geq 0, \\
\Phi_{1}(x) \ell_{1}(x)=(-2+x) x^{3} \leq 0,
\end{gathered}
$$

which is the form of (IVVC) with $n=1, p=q=0$ and $r=1$. The feasible region of (IVVC-2) is

$$
\mathbb{F}_{2}=\left\{x \in R \mid \ell_{1}(x) \geq 0, \Phi_{1}(x) \ell_{1}(x) \leq 0\right\}
$$


For any feasible $x \in \mathbb{F}_{2}$, the corresponding Mond-Weir type dual problem for the primal problem (IVVC-2) is given by

$(\mathrm{IMWDVC}-1) \quad \max \Psi(y)=\left[\Psi^{L}(y), \Psi^{U}(y)\right]=\left[y+y^{3}, y^{5}\right]$

subject to

$$
\begin{gathered}
\lambda^{L} \nabla \Psi^{L}(y)+\lambda^{U} \nabla \Psi^{U}(y)-\eta_{1}^{\ell} \nabla \ell_{1}(y)+\eta_{1}^{\Phi} \nabla \Phi_{1}(y) \\
=\lambda^{L}+3 \lambda^{L} y^{2}+5 \lambda^{U} y^{4}-3 \eta_{1}^{\ell} y^{2}+\eta_{1}^{\Phi}=0, \\
\eta_{1}^{\ell} \geq 0, \quad \text { if } 1 \in \Lambda_{+}, \eta_{1}^{\ell} \in R, \text { if } i \in \Lambda_{0}, \\
\eta_{1}^{\Phi} \leq 0, \quad \text { if } 1 \in \Lambda_{0+}, \eta_{1}^{\Phi} \geq 0, \quad \text { if } 1 \in\left(\Lambda_{0-} \cup \Lambda_{+-}\right), \eta_{1}^{\Phi} \in R, \quad \text { if } i \in\left(\Lambda_{00} \cup \Lambda_{+0}\right), \\
0<\lambda^{L}, \lambda^{U} \in R, \\
-\eta_{1}^{\ell} \ell_{1}(y) \geq 0, \eta_{1}^{\Phi} \Phi_{1}(y) \geq 0 .
\end{gathered}
$$

Let $\mathbb{W}_{2}$ be the set of all feasible solutions of the problem (IMWDVC-1) and note that, $\left(y, \lambda^{L}, \lambda^{U}, \eta_{1}^{\ell}, \eta_{1}^{\Phi}\right)=$ $\left(0, \frac{1}{2}, \frac{1}{2}, 1,-\frac{1}{2}\right)$ is a feasible solution for (IMWDVC-1). Furthermore, it is not difficult to see that $\lambda^{L} \Psi^{L}()+$. $\lambda^{U} \Psi^{U}($.$) is pseudoconvex at y$ on $\mathbb{F}_{2} \cup p r \mathbb{W}_{2}$ and $\ell_{1}(),. \Phi_{1}($.$) are quasiconvex at y$ on $\mathbb{F}_{1} \cup p r \mathbb{W}_{2}$.

Also, for the feasible solutions $x=1$ for $(I V V C$-2) $)$ and $\left(y, \lambda^{L}, \lambda^{U}, \eta_{1}^{\ell}, \eta_{1}^{\Phi}\right)=\left(0, \frac{1}{2}, \frac{1}{2}, 1,-\frac{1}{2}\right)$ for (IMWDVC1), we observe that

$$
\Psi(x)>_{L U} \Psi(y) .
$$

Hence the weak duality Theorem 4.1 is verified.

Theorem 4.2 (Strong Duality). Let $\tilde{x}$ be a LU optimal solution to the problem (IVVC) and the generalized Guignard constraint qualification (IVVC-GCQ) is satisfied at $\tilde{x}$. Then there exist $\tilde{\lambda}^{U}>0, \tilde{\lambda}^{L}>0, \tilde{\mu} \in R_{+}^{p}$, $\gamma \in R^{q}, \eta^{\ell} \in R^{r}$, and $\eta^{\Phi} \in R^{r}$ such that $\left(\tilde{x}, \lambda^{L}, \lambda^{U}, \mu, \gamma, \eta^{\ell}, \eta^{\Phi}\right)$ is a feasible solution for (IMWDVC) and the two objective values are same. Further, if all the assumptions of the Theorem 4.1 are fulfilled, then the point $\left(\tilde{x}, \lambda^{L}, \lambda^{U}, \mu, \gamma, \eta^{\ell}, \eta^{\Phi}\right)$ is a LU optimal solution of (IMWDVC).

Proof. By assumption $\tilde{x}$ is a LU optimal solution for (IVVC) and the generalized Guignard constraint qualification (IVVC-GCQ) is satisfied at this point, then by Theorem 2.1, there exist $\tilde{\lambda}^{U}>0, \tilde{\lambda}^{L}>0, \tilde{\mu} \in R_{+}^{p}$, $\gamma \in R^{q}, \eta^{\ell} \in R^{r}$, and $\eta^{\Phi} \in R^{r}$ such that the conditions (2.1)-(2.6) are satisfied. Thus, $\left(\tilde{x}, \lambda^{L}, \lambda^{U}, \mu, \gamma, \eta^{\ell}, \eta^{\Phi}\right)$ is feasible in (IMWDVC), moreover, the corresponding objective values of (IVVC) and (IMWDVC) are equal. Further, if $\left(\tilde{x}, \lambda^{L}, \lambda^{U}, \mu, \gamma, \eta^{\ell}, \eta^{\Phi}\right)$ is not a LU optimal solution to (IMWDVC), then there exists a feasible solution $\left(\tilde{y}, \lambda^{L}, \lambda^{U}, \mu, \gamma, \eta^{\ell}, \eta^{\Phi}\right)$ for (IMWDVC), such that the following inequality is satisfied

$$
\Psi(\tilde{x})<_{L U} \Psi(\tilde{y})
$$


This is a contradiction to the Theorem 4.1. Hence $\left(\tilde{x}, \lambda^{L}, \lambda^{U}, \mu, \gamma, \eta^{\ell}, \eta^{\Phi}\right)$ is a LU optimal solution to (IMWDVC).

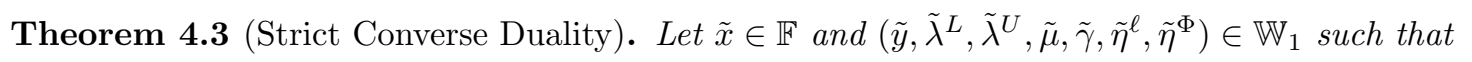

$$
\tilde{\lambda}^{L} \Psi^{L}(\tilde{x})+\tilde{\lambda}^{U} \Psi^{U}(\tilde{x}) \leq \tilde{\lambda}^{L} \Psi^{L}(\tilde{y})+\tilde{\lambda}^{U} \Psi^{U}(\tilde{y})
$$

Further, assume that $\tilde{\lambda}^{L} \Psi^{L}()+.\tilde{\lambda}^{U} \Psi^{U}($.$) is strictly pseudoconvex at \tilde{y}$ on $\mathbb{F} \cup \operatorname{pr} \mathbb{W}_{1}$ and that $\sum_{i=1}^{p} \tilde{\mu}_{i} \varphi_{i}($.$) ,$ $\zeta_{i}().\left(i \in \Lambda_{\zeta}^{+}\right),-\zeta_{i}().\left(i \in \Lambda_{\zeta}^{-}\right),-\ell_{i}().\left(i \in \Lambda_{+}^{+} \cup \Lambda_{0}^{+}\right), \ell_{i}().\left(i \in \Lambda_{0}^{-}\right),-\Phi_{i}().\left(i \in \Lambda_{0+}^{-} \cup \Lambda_{00}^{-} \cup \Lambda_{+0}^{-}\right), \Phi_{i}().(i \in$ $\Lambda_{00}^{+} \cup \Lambda_{0-}^{+} \cup \Lambda_{+0}^{+} \cup \Lambda_{+-}^{+}$) are quasiconvex at $\tilde{y}$ on $\mathbb{F} \cup p r \mathbb{W}_{1}$, then $\tilde{x}=\tilde{y}$.

Proof. Suppose, contrary to the result, that $\tilde{x} \neq \tilde{y}$. Since $\tilde{x}$ and $\left(\tilde{y}, \tilde{\lambda}^{L}, \tilde{\lambda}^{U}, \tilde{\mu}, \tilde{\gamma}, \tilde{\eta}^{\ell}, \tilde{\eta}^{\Phi}\right)$ are feasible solutions in problems (IVVC) and (IMWDVC), respectively, then

$$
\sum_{i=1}^{p} \tilde{\mu}_{i} \varphi_{i}(\tilde{x}) \leq \sum_{i=1}^{p} \tilde{\mu}_{i} \varphi_{i}(\tilde{y})
$$

which by quasiconvexity of $\sum_{i=1}^{p} \tilde{\mu}_{i} \varphi_{i}($.$) at \tilde{y}$ on $\mathbb{F} \cup p r \mathbb{W}_{1}$, we get

$$
(\tilde{x}-\tilde{y})^{T} \sum_{i=1}^{p} \tilde{\mu}_{i} \nabla \varphi_{i}(\tilde{y}) \leq 0
$$

By similar arguments as in Theorem 4.1, we have

$$
\begin{gathered}
(\tilde{x}-\tilde{y})^{T} \nabla \zeta_{i}(\tilde{y}) \leq 0, \forall i \in \Lambda_{\zeta}^{+}, \\
-(\tilde{x}-\tilde{y})^{T} \nabla \zeta_{i}(\tilde{y}) \leq 0, \forall i \in \Lambda_{\zeta}^{-} \\
-(\tilde{x}-\tilde{y})^{T} \nabla \ell_{i}(\tilde{y}) \leq 0, \forall i \in \Lambda_{+}^{+} \cup \Lambda_{0}^{+}, \\
(\tilde{x}-\tilde{y})^{T} \nabla \ell_{i}(\tilde{y}) \leq 0, \forall i \in \Lambda_{0}^{-}, \\
-(\tilde{x}-\tilde{y})^{T} \nabla \Phi_{i}(\tilde{y}) \leq 0, \forall i \in \Lambda_{0+}^{-} \cup \Lambda_{00}^{-} \cup \Lambda_{+0}^{-} \\
(\tilde{x}-\tilde{y})^{T} \nabla \Phi_{i}(\tilde{y}) \leq 0, \forall i \in \Lambda_{00}^{+} \cup \Lambda_{0-}^{+} \cup \Lambda_{+0}^{+} \cup \Lambda_{+-}^{+},
\end{gathered}
$$

which by the definition of index sets one has

$$
(\tilde{x}-\tilde{y})^{T}\left[\sum_{i=1}^{q} \tilde{\gamma}_{i} \nabla \zeta_{i}(\tilde{y})-\sum_{i=1}^{r} \tilde{\eta}_{i}^{\ell} \nabla \ell_{i}(\tilde{y})+\sum_{i=1}^{r} \tilde{\eta}_{i}^{\Phi} \nabla \Phi_{i}(\tilde{y})\right] \leq 0 .
$$

On adding (4.12) and (4.13), we get

$$
(\tilde{x}-\tilde{y})^{T}\left[\sum_{i=1}^{p} \tilde{\mu}_{i} \nabla \varphi_{i}(\tilde{y})+\sum_{i=1}^{q} \tilde{\gamma}_{i} \nabla \zeta_{i}(\tilde{y})-\sum_{i=1}^{r} \tilde{\eta}_{i}^{\ell} \nabla \ell_{i}(\tilde{y})+\sum_{i=1}^{r} \tilde{\eta}_{i}^{\Phi} \nabla \Phi_{i}(\tilde{y})\right] \leq 0
$$

which together with (4.1), implies that

$$
(\tilde{x}-\tilde{y})^{T}\left[\lambda^{L} \nabla \Psi^{L}(y)+\lambda^{U} \nabla \Psi^{U}(y)\right] \geq 0 .
$$


In view of strict pseudoconvexity of $\tilde{\lambda}^{L} \Psi^{L}()+.\tilde{\lambda}^{U} \Psi^{U}($.$) at \tilde{y}$ on $\mathbb{F} \cup p r \mathbb{W}_{1}$, the above inequality gives

$$
\tilde{\lambda}^{L} \Psi^{L}(\tilde{x})+\tilde{\lambda}^{U} \Psi^{U}(\tilde{x})>\tilde{\lambda}^{L} \Psi^{L}(\tilde{y})+\tilde{\lambda}^{U} \Psi^{U}(\tilde{y}),
$$

which contradicts (4.11). This completes the proof of this theorem.

\section{Conclusion}

In this paper, we have derived sufficient optimality conditions for an interesting class of interval-valued optimization problems with vanishing constraints under generalized convexity assumptions. Furthermore, weak, strong and strict converse duality results for a Mond-Weir type dual model have been established. It would be interesting to see whether the results derived in this paper hold for a non-differentiable multiple interval-valued objective programming problems with vanishing constraints. We shall investigate it in our forthcoming papers.

Acknowledgements: This research is financially supported by the King Fahd University of Petroleum and Minerals, Saudi Arabia, under the Internal Project No. IN171012.

Conflicts of Interest: The author(s) declare that there are no conflicts of interest regarding the publication of this paper.

\section{ReferenCES}

[1] J. M. Abadie, On the Kuhn-Tucker theorem, Nonlinear Programming, J. Abadie ed., John Wiley, New York, 1967, pp. 21-36.

[2] W. Achtziger and C. Kanzow, Mathematical programs with vanishing constraints: Optimality conditions and constraint qualifications, Math. Program. 114 (2008), 69-99.

[3] Y. An, G. Ye, D. Zhao and W. Liu, Hermite-Hadamard type inequalities for interval (h1, h2)-convex functions, Mathematics, 7 (2019), Art. ID 436.

[4] M. S. Bazaraa, H.D. Sherali and C.M. Shetty, Nonlinear Programming. Theory and Algorithms. 2nd edition, John Wiley \& Sons, Hoboken, 1993.

[5] M. P. Bendsøe and O. Sigmund, Topology Optimization-Theory, Methods and Applications, 2nd ed., Springer, Heidelberg, Germany, 2003.

[6] A. K. Bhurjee and G. Panda, Efficient solution of interval optimization problem, Math. Method. Oper. Res. 76 (2012), 273-288.

[7] R. W. Cottle, A theorem of Fritz John in mathematical programming, RAND Memorandum RM-3858-PR, RAND Corporation, 1963.

[8] M. Guignard, Generalized Kuhn-Tucker conditions for mathematical programming problems in a Banach space, SIAM J. Control, 7 (1969), 232-241.

[9] A. Khare and T. Nath, Enhanced Fritz John stationarity, new constraint qualifications and local error bound for mathematical programs with vanishing constraints, J. Math. Anal. Appl. 472 (2019), 1042-1077. 
[10] Z.-Q. Luo, J.-S. Pang and D. Ralph, Mathematical Programs with Equilibrium Constraints, Cambridge University Press, Cambridge, 1997.

[11] O. L. Mangasarian and S. Fromovitz, The Fritz John necessary optimality condition in the presence of equality and inequality constraints, J. Math. Anal. Appl. 17 (1967), 37-47.

[12] S. K. Mishra, V. Singh, V. Laha and R. N. Mohapatra, On constraint qualifications for multiobjective optimization problems with vanishing constraints, Optimization Methods, Theory and Applications, Springer Berlin Heidelberg (2015), 95-135.

[13] R. Osuna-Gómez, B. Hernández-Jiménez, Y. Chalco-Cano and G. Ruiz-Garzón, New efficiency conditions for multiobjective interval-valued programming problems, Inform. Sci. 420 (2017), 235-248.

[14] J. V. Outrata, M. Kočvara and J. Zowe, Nonsmooth Approach to Optimization Problems with Equilibrium Constraints, Nonconvex Optimization and its Applications, Kluwer, Dordrecht, 1998.

[15] D. W. Peterson, A review of constraint qualifications in finite-dimensional spaces, SIAM Rev. 15 (1973), 639-654.

[16] A. Sadeghi, M. Saraj and N. M. Amiri, Efficient solutions of interval programming problems with inexact parameters and second order cone constraints, Mathematics, 6 (2018), Art ID 270.

[17] D. Singh, B. A. Dar and A. Goyal, KKT optimality conditions for interval valued optimization problems, J. Nonlinear Anal. Optim. 5 (2014), 91-103.

[18] D. Singh, B. A. Dar and D. S. Kim, KKT optimality conditions in interval-valued multiobjective programming with generalized differentiable functions, European J. Oper. Res. 254 (2016), 29-39.

[19] M. Slater, Lagrange multipliers revisited: a contribution to nonlinear programming, Cowles Commission Discussion Paper, Mathematics, 403, 1950.

[20] I. M. Stancu-Minasian, Stochastic Programming with Multiple Objective Functions, D Reidei Publishing Company, Bordrecht, 1984.

[21] Y. Sun and L. Wang, Optimality conditions and duality in nondifferentiable interval- valued programming, J. Ind. Manage. Optim. 9 (2013), 131-142.

[22] Z. Wang and S. C. Fang, On constraint qualifications: motivation, design and inter-relations, J. Ind. Manage. Optim. 9 (2013), 983-1001.

[23] L. Wang, G. Yang, H. Xiao, Q. Sun, J. Ge, Interval optimization for structural dynamic responses of an artillery system under uncertainty, Eng. Optim. 52 (2020), 343-366.

[24] H. C. Wu, On interval valued nonlinear programming problems, J. Math. Anal. Appl. 338 (2008), 299-316.

[25] H. C. Wu, The Karush Kuhn Tuker optimality conditions in multiobjective programming problems with interval-valued objective functions, European J. Oper. Res. 196 (2009), 49-60.

[26] H. C. Wu, Solving the interval-valued optimization problems based on the concept of null set, J. Ind. Manage. Optim. 14 (3) (2018), 1157-1178. 